\title{
The global optimization of Morse clusters by potential energy transformations
}

\author{
J. P. K. Doye • R. H. Leary • M. Locatelli • F. Schoen \\ University Chemical Laboratory, Lensfield Road, Cambridge CB2 1EW, United Kingdom \\ San Diego Supercomputer Center, University of California, San Diego, USA \\ Dipartimento di Informatica, Università di Torino, Corso Svizzera 185, I-10149 Torino, \\ Italy \\ Dipartimento di Sistemi e Informatica, Università di Firenze, Via di S.Marta, 3, I-50139 \\ Firenze 185, Italy \\ jpkd1@cam.ac.uk•leary@sdsc.edu•locatell@di.unito.it•schoen@ing.unifi.it
}

The Morse potential is a simple model pair potential that has a single parameter $\rho$ which determines the width of the potential well and allows a wide variety of materials to be modelled. Morse clusters provide a particularly tough test system for global optimization algorithms, and one that is highly relevant to methods that are to be applied to find the optimal configuration of a biomolecule. In particular, large $\rho$ values are very challenging and, until now, no unbiased global optimization method has been able to detect all the (putative) global minima at $\rho=14$ for clusters with up to $N=80$ atoms. In this paper we introduce some techniques for transforming the original Morse potential that allow us to considerably increase the efficiency in locating the known global minima and also to discover some new optimal clusters. These methods are promising candidates for application to the optimization of biomolecules.

(Morse potential; global optimization; basin-hopping; two-phase local search)

\section{Introduction}

Test problems have played an important role in the field of global optimization, aiding the development of new algorithms by providing a means to benchmark their efficiency [11]. Lennard-Jones clusters have become just such an archetypal test problem for global optimization algorithms that specialize in finding the lowest energy 'molecular' configuration, be it a cluster, a biomolecule or a crystal [30], with probably hundreds of published applications to this problem. There is probably no equivalent test system for the protein folding 
problem, at least when modelled in a continuous configuration space, ${ }^{1}$ and many of the most successful algorithms for Lennard-Jones clusters have been subsequently applied to optimize polypeptides and proteins.

The choice of Lennard-Jones clusters as a test system is for a number of reasons. The Lennard-Jones potential is extremely simple. Excellent putative global minima have now been tabulated for clusters with up to 309 atoms [28, 29]. Lennard-Jones clusters have also become a model system for understanding a wide range of cluster properties, such as their structure, thermodynamics, dynamics and energy landscapes, allowing the ease or difficulty of global optimization to be correlated with these cluster properties [8].

However, Lennard-Jones clusters have a number of deficiencies as a test system. Firstly, they are rather structurally uniform. Virtually all of the global minima in the relevant size range are based on Mackay icosahedra [25]. Secondly, for systems of their dimensionality Lennard-Jones clusters are relatively easy to optimize. This is because the potential energy landscapes for these systems give rise to strong funnelling properties, directing the system down towards the global minimum [6]. For example, even though there are an estimated $10^{21}$ geometrically distinct minima for a 55-atom Lennard-Jones cluster, the basin-hopping algorithm is able to find the global minimum from a random starting point after only sampling on average 150 of those minima [30].

The most difficult Lennard-Jones clusters to optimize occur at the few sizes where the global minimum has a structure that is not in fact based on the Mackay icosahedron. In these cases, the energy landscape has a double-funnel topography, where the dominant funnel leads down to icosahedral structures and there is a second narrower, and less accessible, funnel leading down to the global minimum [6]. Typically, these examples are one or two orders of magnitude more difficult [15]. That a few examples are the most meaningful tests of the performance of an algorithm is probably not the behaviour one wants from a test system. Rather it is much better to have a more uniform difficulty.

Because of these deficiencies, Morse clusters have been suggested as a much more rigorous alternative test system [7]. The Morse pair potential [23] as a function of the interatomic distance $r$ between two atoms is defined as

$$
v_{\rho}(r)=[\exp \{\rho(1-r)\}-1]^{2}-1,
$$

\footnotetext{
${ }^{1}$ For proteins modelled on a lattice the archetypal test system is probably the HP model [14]. However, global optimization techniques for systems in discrete and continuous spaces have substantial differences.
} 
and so a $N$-atom Morse cluster, $\mathrm{M}_{N}$, has a potential energy given by

$$
\operatorname{Morse}_{N}(X ; \rho)=\sum_{i<j} v_{\rho}\left(\left\|X_{i}-X_{j}\right\|\right) \text {. }
$$

Here $X$ is the $3 N$-dimensional vector of atomic coordinates, and $X_{i}$ is the 3 -dimensional vector of coordinates of the $i$-th atom. The potential has a single parameter, $\rho$, that determines the width of the well (Fig. 1), thus allowing it to capture aspects of the interactions for a wide range of materials. For example, the Lennard-Jones potential has the same curvature at the bottom of the well when $\rho=6$; for this value of $\rho$ the structural behaviour of the two systems is very similar. Approximating the interactions between $\mathrm{C}_{60}$ molecules by a pair potential gives $\rho=13.6$, whereas appropriate values for interactions between alkai metals are typically about $\rho=3.1$.

The Morse clusters show a wide variety of structural behaviour as a function of $\rho$. As $\rho$ is increased the structures of the global minima change from polytetrahedral to Mackay icosahedral to decahedral to close-packed [7]. The potential energy landscape also changes character with $\rho$. Firstly, as $\rho$ is increased the number of minima increases rapidly, changing the landscape from smooth to rough [21]. On this basis alone, one expects the difficulty of global optimization to increase with $\rho$. Therefore, from the global optimization perspective $\rho<6$ is the less interesting regime, since optimization is easier than for Lennard-Jones clusters.

Secondly, the energy landscape is much more likely to have a multiple-funnel character at larger values of $\rho$ [9], further increasing the difficulty of global optimization. Usually, there are a variety of low-energy configurations, mainly decahedral and close-packed, that are structurally very different and so well-separated in configuration space. This feature makes Morse clusters especially relevant to biomolecules, where, although there is an evolutionary pressure to make the energy landscapes of proteins sufficiently funnel-like that the protein can fold in a biologically appropriate time scale [3], it is still common to find structures that act as traps hindering the folding process, both in experiment $[13,26]$ and simulations $[1,12]$. Analyses of energy landscapes show that such traps correspond to additional funnels on the energy landscape [16, 17, 22, 24].

The combination of these effects makes the global optimization of Morse clusters at $\rho=10$ and 14 a very challenging task, and one which is orders of magnitude more difficult than Lennard-Jones clusters. A database of putative Morse cluster global minima is available up to $N=80$ [4]. This was obtained through a combination of the application of global 


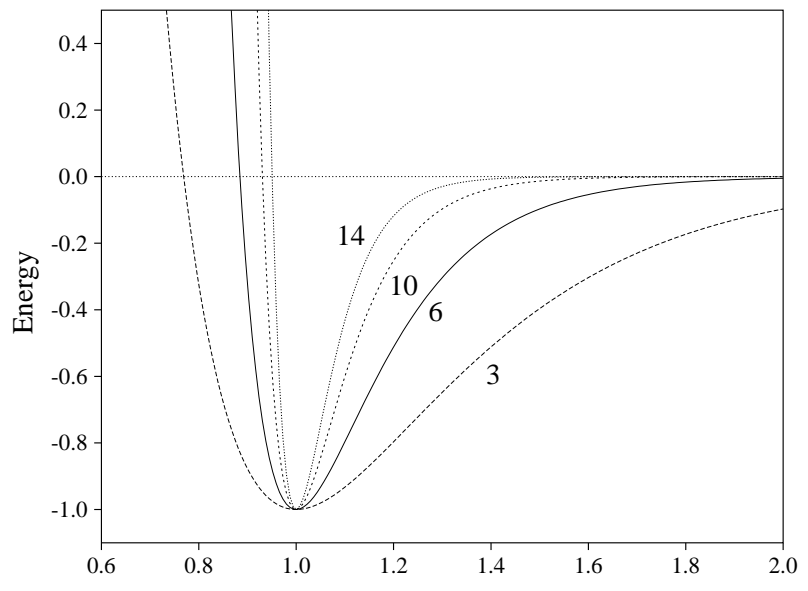

Figure 1: The Morse potential for different values of $\rho$, as labelled.

optimization algorithms and the generation of candidate structures on the basis of structural insight into the problem [7]. It has since been used as a global optimization test system [27], but, until now, no unbiased global optimization algorithm has managed to obtain all the global minima. In this paper, we attempt just such a task.

\section{Basin-Hopping and Funnel Landscapes}

The landscape of a smooth potential function $E(X)$ with many local minima such as the Morse energy function can be viewed as a collection of basins of attraction, each associated with a local minimum. A gradient-based local optimization procedure such as a steepest descent, Newton, or quasi-Newton algorithm, when initiated at a point in a given basin of attraction, will converge to the associated local minimum. One strategy for the global minimization of such energy functions is to combine a standard gradient-based local search method for finding local minima with a global method for searching over the various basins of attraction. This general approach has come to be known as 'basin-hopping' [29].

Basin-hopping algorithms typically search the space of local minima by moving between adjacent local minima across a common basin boundary. This search strategy can be particularly effective on landscapes where the local minima are organized into a few large-scale funnels [15]. A funnel consists of a collection of local minima such that all monotonically descending sequences of successively adjacent local minima entering the funnel terminate at the funnel bottom. Note that all monotonically descending sequences must eventually enter a funnel, and clearly a downwardly biased search over adjacent local minima is a reasonable 
strategy to locate a funnel bottom. Note also that the global minimum lies in the set of funnel bottoms.

For any given point $X$ and local search procedure $L S$, let $L S(X)$ be the local minimum of the energy function $E(X)$ that is obtained by applying $L S$ to the starting point $X$. A typical basin-hopping algorithm produces a sequence of local minima from the iteration

$$
X^{[i+1]}=L S\left(X^{[i]}+S\right)
$$

where $S$ is a random perturbation used to escape the current basin of attraction. The size of $S$ is controlled to reach only nearby (and usually adjacent) local minima. Additionally, an acceptance/rejection step that depends on the energy change $E\left(X^{[i+1]}\right)-E\left(X^{[i]}\right)$ is applied to bias the search toward lower energy values. Thus the sequence $\left\{X^{[i]}\right\}$ can be viewed as a Markov chain on a finite state space consisting of the local minima of $E(X)$. The transition probabilities are determined by the distribution of the perturbations $S$ as well as the details of the acceptance/rejection rule.

In this paper we consider three forms of basin-hopping:

i) $\mathrm{BH}$, the original basin-hopping algorithm ${ }^{2}$ of Wales and Doye [29]. The point $X^{[i+1]}$ is accepted or rejected according the Metropolis criterion, that is, all downhill steps $\left(E\left(X^{[i+1]}\right)<\right.$ $\left.E\left(X^{[i]}\right)\right)$ are accepted, and uphill steps $\left(E\left(X^{[i+1]}\right)>E\left(X^{[i]}\right)\right)$ are accepted randomly with probability

$$
p=e^{\left(E\left(X^{[i+1]}\right)-E\left(X^{[i]}\right)\right) / T}
$$

Here $T>0$ is a preselected 'temperature' parameter that remains fixed (i.e., no annealing is done) throughout a given run. If the step is rejected, new random perturbations $S$ are generated until a step is accepted. Starting from a random initial local minimum, the Markov chain is extended for as long as desired (usually at least several thousand steps), and there are usually no absorbing states if the distribution of $S$ is properly selected. A putative global minimum is the lowest energy state visited.

ii) MSBH (Monotonic Sequence Basic-Hopping), a variant due to Leary [15] that attempts to exploit the funnel structure of the landscape more aggressively than BH. MSBH is basically $\mathrm{BH}$ at a temperature $T=0$, that is, only downhill steps are accepted, so the sequence of

\footnotetext{
${ }^{2}$ The same algorithm was developed independently in the protein folding community and called Monte Carlo with minimization [18].
} 
states is monotonically descending in energy. Once the sequence enters a given funnel, it will proceed on an energetically descending path to the funnel bottom, which is an absorbing state in the Markov chain. After a funnel bottom is recognized by the lack of progress in a sufficiently large number of attempted moves, the Markov chain is restarted from a new randomly selected local minimum. MSBH can be viewed as a multistart approach that samples funnel bottoms. It can be particularly effective if the number of funnel bottoms is small compared to the number of local minima, and the probability for entering the correct funnel associated with the global minimum is reasonably large.

iii) TP-MSBH (Two-Phase MSBH), due to Locatelli and Schoen [20], who introduce a modified energy function

$$
E_{G}(X)=E(X)+G(X)
$$

where $G(X)$ is a geometrically-inspired penalty term. Let $L S_{G}(X)$ be the local minimum of $E_{G}(X)$ obtained by initiating a local search procedure at $X . L S_{G}(X)$ is then used as the starting point for a local search of $E(X)$, that is,

$$
X^{[i+1]}=L S\left(L S_{G}\left(X^{[i]}+S\right)\right)
$$

Thus two local searches, one on the landscape of $E_{G}(X)$ and a second on the landscape of $E(X)$, are performed at each step (see Section 3 for more details). As in MSBH, the step is accepted if it improves the energy $E(X)$, and otherwise a new perturbation is attempted. The entire procedure is restarted from a new random local minimum of $E(X)$ after a prescribed number of step attempts with no progress. The effect of the first phase local optimization on the landscape of $E_{G}(X)$ is to bias the search toward local minima of $E(X)$ with specific shape-related geometrical properties. As seen below, this can greatly increase the probability of entering the proper funnel and finding the global minimum.

Wales and Doye [29] note that basin-hopping algorithms for the global minimization of $E(X)$ in effect search a transformed piecewise constant 'plateau' energy function $P(X)=$ $E(L S(X))$ where the the landscape of $P(X)$ consists of interlocking plateaus, each covering a given basin of attraction at a height equal to the energy of the local minimum in the basin. Much of the power of basin-hopping methods derives from the fact that, unlike global search methods applied directly to $E(X)$, there are no energetic barriers for moving downhill between plateaus, and basin crossing can easily take place at any point along a 
basin boundary with no necessity to search for special boundary crossing points such as first order transition points.

\section{Two-Phase local searches}

In this section Two-Phase (TP) local searches, already introduced in Section 2, are described in more detail. The formal scheme of TP local searches is the following.

Procedure: TwoPhaseLocOpt $(U)$;

Phase I: find

$$
Y=\left[Y_{1}, \ldots, Y_{N}\right]=\arg \operatorname{local} \min \operatorname{Morse}_{N}^{\bmod }(X)
$$

using $U=\left[U_{1}, \ldots U_{N}\right] \in R^{3 N}$ as the starting point;

Phase II: find

$$
Z=\left[Z_{1}, \ldots, Z_{N}\right]=\arg \text { local min } \operatorname{Morse}_{N}(X ; \rho)
$$

using $Y$ as a starting point;

end: $\operatorname{return}(Z)$.

Given a starting point $U$, the first phase returns a local minimum of a modified potential Morse ${ }_{N}^{\text {mod }}$, while in the second phase such local minimum becomes the starting point of a local search driving to a local minimum of the original potential. While the second phase has the important task of driving the search back to the space of local minima of the original potential, the first phase can considerably increase the speed at which optimal clusters are detected in particular through the choice of a modified potential that allows the funnel structure of the landscape to be modified in such a way that the funnel containing the global minimum is favoured with respect to the others [5]. Therefore, the efficiency of TP local searches strongly depends on the modified potential. In this paper we employ the following modified potential (see also [19], [20]):

$$
\begin{aligned}
\operatorname{Morse}_{N}^{\bmod }(X)= & \min \sum_{i<j}\left(v_{\rho^{\prime}}\left(\left\|X_{i}-X_{j}\right\|\right)\right. \\
& +\mu\left\|X_{i}-X_{j}\right\| \\
& \left.+\left(\max \left\{0,\left\|X_{i}-X_{j}\right\|_{W}^{2}-D^{2}\right\}\right)^{2}\right)
\end{aligned}
$$


where

$$
\left\|X_{i}-X_{j}\right\|_{W}=\left(\sum_{k=1}^{3} w_{k}\left(X_{i}^{k}-X_{j}^{k}\right)^{2}\right)^{\frac{1}{2}}
$$

is the weighted distance between two atoms. Note that $\rho^{\prime}, \mu, D$ are nonnegative parameters, while $w_{1}=1$ and $w_{2}, w_{3} \in[0,1], w_{2} \geq w_{3}$. In the following, we comment on the different terms of the modified potential and the different impact that such terms have on the performance of TP-MSBH. Term (2) is the original Morse potential but with a parameter $\rho^{\prime}$ which is possibly different with respect to $\rho$. Parameter $\rho^{\prime}$ allows to control the degree of smoothness of the modified potential: as $\rho^{\prime}$ increases the landscape of the modified potential becomes rougher and rougher and the number of local minima increases. The computations show that the choice $\rho^{\prime}=\rho$ is often quite appropriate, although in some cases there may be some advantage from using $\rho^{\prime} \neq \rho$.

Term (3) is a linear penalization of distance between atoms (typical values for $\mu$ range between 0 and 0.5). Its effect is that of disfavouring clusters containing atom pairs at large distance, thus favouring compactness of the clusters. While the use of this term gave some benefits during the optimization of Lennard-Jones clusters (see [20]), its relevance for the optimization of Morse clusters with large $\rho$ values is less obvious and, as we will see, in the computations, setting $\mu=0$ is often a feasible choice.

Term (4) is undoubtedly the most relevant one. It is a weighted diameter penalization term, i.e. it strongly penalizes large weighted distance between atoms, more precisely weighted distance significantly larger than $D$ (parameter $D$ is often chosen between one or two units smaller than the expected diameter of the cluster, whose increase is proportional to $N^{1 / 3}$ and whose estimated value is returned by appropriate formulae). It also favours compact structures but whose shapes can be very different according to the choice of parameters $w_{2}$ and $w_{3}$. Indeed, if $w_{2}=w_{3}=1$, then we are favouring spherical shapes, but as we decrease one of or both the weights $w_{2}$ and $w_{3}$ we favour more oblate or prolate shapes. The advantages of using different weights $w_{2}$ and $w_{3}$ were already noticed in some cases for the optimization of Lennard-Jones clusters (see [20]) but for the very difficult Morse clusters with large $\rho$ values the advantages are much more evident because of the larger variability of the shapes of these clusters. An important question about parameters $w_{2}$ and $w_{3}$ is the following: are there unique values for the weights $w_{2}$ and $w_{3}$ which are appropriate for all the Morse clusters? Unfortunately, the answer is no. As it will become clear in Section 4, weights $w_{2}$ and $w_{3}$ which are extremely successful for some $N$ values, completely fail for 
other $N$ values. The optimal choice of $w_{2}$ and $w_{3}$ for a given $N$ value seems to be related to the moments of inertia of the corresponding optimal cluster (see Section 5) and these moments vary considerably with $N$ for Morse clusters with large $\rho$ value. This may seem to be bad news because we, of course, do not know optimal clusters in advance and, therefore, we cannot choose the best values for $w_{2}$ and $w_{3}$. However, in fact TP-MSBH appears to be a quite robust method with respect to the choice of $w_{2}$ and $w_{3}$. Indeed, even choices that are not too close to the moments of inertia often allow us to detect the optimal clusters very efficiently. In spite of the large variability of the moments of inertia, the computational results in Section 4 will show that almost all optimal Morse clusters at $\rho=14$ for $N$ up to 80 can be detected by TP-MSBH coupled with Forward/Backward procedures using only five different sets of parameters, often much more efficiently than with MSBH.

\section{Computational results}

The first extensive computations of global minima of Morse clusters were described in [7] and made available on the WWW in the Cambridge Cluster Database (CCDB) [4]. These included putative global minima for $N \leq 80$ and $\rho=3,6,10,14$. The global minima were found by a combination of the BH algorithm and direct construction guided by the authors' experience with global minima of the Morse and other potentials. BH by itself was able to find all the initial results for $\rho=3,6$, and 10, and all but six for $\rho=14$. Later improved global minima for $N=61$ and $68, \rho=10$ (61B' and 68D' in the CCDB) were found by W. Branz and noted in [9] and for $N=30, \rho=14$ (30D') by D. J. Wales using BH.

The MSBH algorithm was applied in the current study and found all the $\rho=3,6$, and 10 cases, including 61B' and 68D', and most of the $\rho=14$ cases, including 30D'. MSBH also discovered the new 78D', $\rho=6$ 'double icosahedron' putative global minimum. The $\rho=3$ cases proved to be far easier than comparably sized Lennard-Jones (LJ) clusters, while the $\rho=6$ cases were of similar difficulty. However, particularly for $N>40, \rho=10$ and 14 , a few cases required extraordinary computational effort as measured by a number of trials (monotonic sequence starts from a random initial state) almost two orders of magnitude more than the most dificult Lennard-Jones clusters in the same size range. All of the LennardJones optimal clusters were found with MSBH within 1000 trials [15], whereas quite a few of the Morse cases for the larger values of $\rho$ required more than 1000 trials for a single hit. In five 
Table 1: The five sets of parameters employed in the TP-MSBH computations. The dependence of the parameter $D$ on cluster size is given in parentheses.

\begin{tabular}{||c||c|c|c|c|}
\hline & $D$ & $\mu$ & $w_{2}$ & $w_{3}$ \\
\hline$P 1$ & $3.5(N \leq 60)-4$ & 0.1 & 1 & 1 \\
P2 & $3(N \leq 60)-3.5$ & 0 & 1 & 0.7 \\
P3 & 3 & 0 & 0.8 & 0.8 \\
P4 & 2.5 & 0 & 0.75 & 0.5 \\
P5 & $2(N \leq 60)-2.5$ & 0 & 0.5 & 0.5 \\
\hline
\end{tabular}

instances for the larger clusters at $\rho=14$, current putative Morse global minima have not yet been obtained by MSBH. Thus MSBH falls slightly short of meeting the challenge implicit in the observation in the CCDB that no unbiased global optimization method has been able to find all of the current putative global minima for $N \leq 80$ for the four $\rho$ values. Here unbiased means not incorporating detailed foreknowledge of the properties and morphological forms of known Morse global minima. Examples of biased algorithms include searching over highly restricted domains such as fcc, Mackay icosahedral, or Marks decahedral lattices [25, 28], which would necessarily preclude hitting any global minima outside those domains, and seeding algorithms with structures likely to be related to the global minima being sought [31].

In the remainder of this section we present and comment on computational results obtained using TP-MSBH, which show that algorithm easily meets the CCDB challenge. For economy of presentation, we restrict attention here primarily to $41 \leq N \leq 80$ and $\rho=14$, the most difficult cases for MSBH. However, we have successively applied TP-MSBH to all the remaining cases covered in the CCDB, as well as some intermediate values of $\rho$ for which putative global minima structures are given in the CCDB but explicit global optimizations have not previously been performed. The modified Morse function (see Section 3, eqs. 2-4) depends on a number of adjustable parameters, the most important of which are the diameter penalty weights $w_{1}, w_{2}, w_{3}$ and the diameter threshold $D$. Here we fix $\rho^{\prime}=\rho$, and $\mu=0$, except when using the spherically symmetric weight set with all weights equal to 1 , in which case we set $\mu=0.1$ (the use of $\mu$ is not strictly necessary, but we have found the fixed setting $\mu=0.1$ to be more useful than not in the spherically symmetric case). As discussed in Section 3, good ranges for $D$ can be found systematically from $N$, so the diameter penalty weights are the primary parameters of interest.

Our testing has shown that usually a significant range of diameter penalty weights are effective for a given case, and a close match to an 'optimal' set of weights is not necessary 
Table 2: Number of successes in 50 random runs for the five different parameter sets.

\begin{tabular}{||c||c|c|c|c|c|c||c||c|c|c|c|c|c||}
$N$ & $P 1$ & $P 2$ & $P 3$ & $P 4$ & $P 5$ & $F B$ & $N$ & $P 1$ & $P 2$ & $P 3$ & $P 4$ & $P 5$ & $F B$ \\
41 & 0 & 0 & 0 & 2 & 0 & & 61 & 0 & 0 & 0 & 0 & 0 & \\
43 & 0 & 0 & 0 & 0 & 0 & $f$ & 62 & 0 & 4 & 0 & 0 & 0 & \\
44 & 0 & 0 & 0 & 1 & 0 & & 63 & 0 & 21 & 0 & 25 & 0 & \\
45 & 0 & 0 & 0 & 0 & 0 & $f$ & 64 & 1 & 18 & 0 & 26 & 0 & \\
46 & 0 & 0 & 0 & 0 & $b$ & 65 & 0 & 6 & 0 & 3 & 0 & \\
47 & 0 & 0 & 0 & 0 & 3 & & 66 & 0 & 8 & 0 & 0 & 0 & \\
48 & 0 & 0 & 0 & 1 & & 67 & 0 & 3 & 0 & 0 & 0 & \\
49 & 0 & 0 & 0 & 4 & 28 & & 68 & 5 & 0 & 0 & 0 & 0 & \\
50 & 0 & 0 & 29 & 14 & & 69 & 0 & 1 & 8 & 1 & 0 & \\
51 & 47 & 0 & 5 & 0 & & 70 & 0 & 7 & 5 & 7 & 0 & \\
52 & 0 & 31 & 0 & 13 & 0 & & 71 & 3 & 2 & 49 & 12 & 0 & \\
53 & 1 & 4 & 1 & 0 & 0 & & 73 & 1 & 0 & 25 & 1 & 0 & \\
54 & 0 & 0 & 0 & 0 & 0 & $b$ & 74 & 2 & 0 & 47 & 18 & 36 & \\
55 & 0 & 0 & 0 & 0 & 0 & $b$ & 75 & 11 & 0 & 44 & 8 & 5 & \\
56 & 1 & 0 & 0 & 0 & 0 & & 76 & 2 & 0 & 38 & 3 & 6 & \\
57 & 1 & 0 & 0 & 0 & 0 & & 77 & 0 & 0 & 11 & 10 & 11 & \\
58 & 39 & 1 & 12 & 0 & 0 & & 78 & 0 & 0 & 0 & 5 & 4 & \\
59 & 39 & 0 & 19 & 0 & 0 & & 79 & 1 & 0 & 0 & 0 & 0 & \multirow{2}{*}{$(1$} \\
60 & 25 & 0 & 20 & 0 & 0 & & 80 & 0 & 0 & 0 & 0 & 0 & $f$ \\
\hline
\end{tabular}

for successful application of the TP-MSBH algorithm. Thus a very general and effective algorithm can be formulated by simply spreading a sufficiently large number of weight sets fairly uniformly over the relevant portions of weight space so that at least one set is likely to be effective. Since the weight space is of low dimension (in effect, 2-dimensional since $w_{1}$ is fixed at 1.0) and the relevant region $0.0 \leq w_{3} \leq w_{2} \leq 1.0$ quite small, the algorithm can effectively sample weight space with a manageable number of weight sets.

After a very limited amount of experimentation, we found that the five sets of parameters P1-P5 given in Table 1 were sufficient to find all but one $(N=61)$ of the cases of interest using 50 trials for each of the parameter sets. The results are reported in Table 2, where each column P1-P5 reports the number of successes in 50 random runs of TP-MSBH with the given set of parameters. For the sizes where the optimal cluster has not been detected with any of the five set of parameters, column FB reports a $f$ if the optimal cluster has been detected by a forward procedure and a $b$ if it has been detected by backward procedure from a putative global minimum found at an adjacent size with the same parameter set. The forward/backward procedures are commonly used in cluster optimization studies to take 
advantage of the fact that the global optima for adjacent size $N$ and $N+1$ sometimes (but by no means always) have very similar structures, differing only in the placement of usually one and at most a few atoms, typically on the surface. Thus the removal or addition of an atom at one size, in conjunction with some simple searches, is a useful technique for locating global minima at the adjacent sizes. Here we limit the procedure to 100 random additions/deletions of a single atom to/from the best configuration with $N-1 / N+1$ atoms found by TP-MSBH. For the sake of completeness we point out that the optimal configurations found by the forward/backward procedures (those for $N=42,44,45,54,55,80$ ) can also be found directly by TP-MSBH by simply increasing the number of random trials (never more than 1000 and usually much less).

Along with the 'obvious' choice of the spherically symmetric weight set $w_{1}=w_{2}=w_{3}=$ 1.0 in $\mathrm{P} 1$, the remaining four weight sets in P2 - P5 were selected primarily on the basis of broad coverage of the weight space (see Fig. 2 in the next section) rather than by tuning for specific results. The five sets of parameters employed here are not unique or the end product of an extensive parmeter selection and optimization process, and certainly others with good coverage of the weight space will work as well. Also, filling in gaps in the weight space coverage of P1-P5 will give improved performance. For example, the single missed case at $N=61$ is easily found in 2 of 50 trials using a weight set P6 midway between P3 and P5. Also, the choice $w_{2}=0.6, w_{3}=0.4, D=2$ in an area of weight space not well covered by P1-P5 allows us to detect all optimal clusters for $N=41-47$ with much higher success rates and without the use of forward/backward procedures. However, we emphasize our aim is not to find optimal parameters for each $N$ value but to show that a very limited number of parameter sets is sufficient to find all optimal clusters and that an efficient and robust algorithm can be constructed without excessive parameter tuning or foreknowledge of the global minima being sought.

We have confirmed the effectiveness, efficiency and robustness of the TP-MSBH approach proposed here by easily finding all previously known optimal clusters in the CCDB. Further evidence of these properties is provided by the discovery or rediscovery by TS-MSBH of several new putatively optimal clusters with improved energies relative to those known prior to the current study. For $N=78$ at $\rho=6$ a new optimal cluster was first discovered by MSBH, and later also found with a much higher success rate by TP-MSBH. For $N=$ $24,25,45,48,51$ at $\rho=8$, new optimal clusters have been discovered by TP-MSBH. All these new clusters are shown and described in Section 6. 
Several of the cases $N=41-80, \rho=14$ discussed in this section have proven to be enormously difficult for the $\mathrm{BH}$ and $\mathrm{MSBH}$ algorithms. For more than half of the cases $N>60$, 1000 trials of the MSBH algorithm has proven to be insufficient, and several required more than 10,000 trials to achieve a single success. In five cases, neither version of single phase basin-hopping to date has been successful despite extensive computational effort. In contrast, the TP-MSBH algorithm has consistently found all the global minima with only a few hundred trials at each $N$ value spread over a very limited number of parameter sets selected for broad coverage of the parameter space. Thus the power and generality of the two phase idea with a weighted diameter penalization is demonstrated by the reliably large amplification relative to single phase approaches of the probability of hitting the global minimum with at least one parameter set. This far outweighs the dilutive effect of distributing a given number of trials over multiple parameter sets.

\section{Shape Factors and Diameter Penalty Weights}

The shape of a cluster can be approximately described by shape factors representing the ratios of the lengths of the minor, intermediate, and major axes of a ellipsoid with the same principle moments of inertia. The 3 by 3 moment of inertia tensor (also known as the shape tensor or radius of gyration tensor) for any configuration $X$ of $N$ identical atoms is defined as

$$
Q_{m n}=\frac{1}{N} \sum_{j>i}\left(X_{i}^{m}-X_{j}^{m}\right)\left(X_{i}^{n}-X_{j}^{n}\right)
$$

The eigenvalues $0 \leq \lambda_{1} \leq \lambda_{2} \leq \lambda_{3}$ of $Q$ correspond to the squared lengths of the equivalent ellipsoid axes, with the eigenvectors representing the directions of the axes. The trace $\operatorname{Tr}(Q)=\lambda_{1}+\lambda_{2}+\lambda_{3}$ is the squared radius of gyration, i.e., the mean square distance of the atoms from the center of mass.

The weighted diameter penalization term for any pair $(i, j)$ of atoms is an increasing function of the quadratic form

$$
\left\|X_{i}-X_{j}\right\|_{W}^{2}=\sum_{k=1}^{3} w_{k}\left(X_{i}^{k}-X_{j}^{k}\right)^{2}
$$

defined by the weights $1=w_{1} \geq w_{2} \geq w_{3} \geq 0$. The corresponding ellipsoids of constant weighted diameter penalty are centered at the center of mass of the atom pair with principal axes of length proportional to $1 /\left(w_{k}\right)^{\frac{1}{2}}$ and directions along the coordinate axes. 
Suppose the eigenvalues of the moment of inertia tensor of the global minimum are known in advance, and we wish to find diameter penalty weights that will work well in the TP-MSBH algorithm to find that global minimum. The obvious choice $w_{1}=1, w_{2}=\lambda_{1} / \lambda_{2}, w_{3}=\lambda_{1} / \lambda_{3}$ matches the shape of the ellipsoids of constant weighted diameter penalty to that of the moment of inertia ellipsoid, thus penalizing configurations with the 'wrong' shape. Examples of moment of inertia weights $\left(w_{2}, w_{3}\right)$ are plotted in Fig. 2 for the cases $N=41-80, \rho=14$, along with the weight sets P1 - P5 described in Section 4. The point $(1,1)$ corresponds to spherical shapes, the bounding line $w_{2}=w_{3}$ to oblate shapes, and the bounding right edge $w_{2}=1$ to prolate shapes.

Empirically we have found that moment of inertia penalty weights are always a reasonable choice in the sense that all global minima have been found within 1000 runs (and usually far fewer) with their use. Moreover they are often very good or even near optimal in terms of approximately maximizing the probability of hitting the global minimum, and in difficult cases, the success of TP-MSBH depends on a reasonable match of the diameter penalty weights to the optimal cluster shape. Conversely, a relatively few weight sets such as P1 - P5 that, as seen in Fig. 2, are fairly uniformly distributed over the space of moment of inertia shape factors, are sufficient to find all the global minima.

An example of the efficacy of matching penalty weights to moment of inertia shape factors is the new double icosahedral global minimum for $N=78, \rho=6$ that was first found by MSBH. This case is in fact quite difficult for that algorithm, which hit it only 3 times in 1000 runs. Using the moment of inertia weights $w_{1}=1.0, w_{2}=0.9718, w_{3}=0.4957$ corresponding to the rather extreme prolate shape of this structure, the TP-MSBH algorithm hit it in 410 of 1000 runs. Weight sets that are a poor match were far less successful. Similarly, exploration of the weight space for the relatively difficult $N=67, \rho=14$ case found the best weights attempted to be $w_{1}=1.0, w_{2}=1.0, w_{3}=0.7$, in excellent agreement with the moment of inertia weights $w_{1}=1.0, w_{2}=0.9986, w_{3}=0.6907$ for this prolate shape. Also, the oblate weight set P3 is the most successful of the five sets discussed in Section 4 for the oblate Marks decahedron at $N=75, \rho=14$. Finally, extremely aspherical structures such as the $N=41-47, \rho=14$ cases where the global minima have very flat decahedral shapes can be among the most difficult for TP-MSBH using just the parameter sets P1-P5. The only successful weight sets P4 and P5 for these cases are the best matches to the moment of inertia weights. However, as mentioned in the previous section, considerably improved results can be obtained with the addition of a weight set closer to the shape factors for theses cases. 


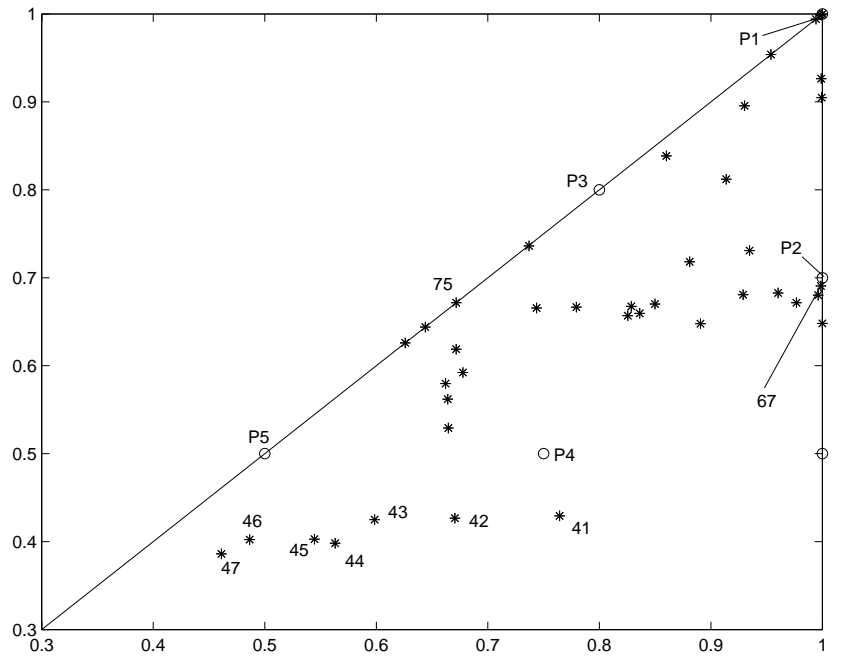

Figure 2: Moment of inertia weights $w_{2}$ (horizontal axis) and $w_{3}$ (vertical axis) for $N=$ $41-80, \rho=14$, are plotted as points ${ }^{~}{ }^{*}$ ' with coordinates $\left(w_{2}, w_{3}\right)$. Weight sets P1 - P5 used to find all global minima are plotted as points 'o'. Cases discussed in the text are labelled with the corresponding $N$ value.

\section{New global minima}

The new global minima that were discovered during this research program are depicted in Figure 3. All these new global minima have been added to the Cambridge Cluster Database $[4]$.

The 24-atom structure is formed from the 23-atom triple icosahedron by the addition of a capping atom at the edge of the cluster. The $\rho=6$ global minimum has the capping atom at the other alternative 4-fold coordinate site. In the 23-atom triple icosahedron, mentioned above, the centres of the three interpenetrating icosahedra form an equilateral triangle. The new $\mathrm{M}_{25}$ global minimum also consists of three interpenetrating icosahedra, but with their centres in an open 'V'-shape. This structure has previously been found for clusters interacting with the Dzugutov potential [10].

The 45-, 48- and 51-atom clusters are all incomplete Mackay icosahedra, but which have a slightly different choice of vacant surface sites compared to the icosahedral global minima previously located at these sizes. In particular, all three structures can be formed from the icosahedral global minimum for the preceding size by the addition of an atom to a vertex site that is only five coordinate, rather than to the edge site that completes the five-fold ring around that vertex. 
At $N=87$ a structure can be formed that is made up of two interpenetrating Mackay icosahedra and is related to the 19-atom double icosahedron in the same way that the 55 -atom Mackay icosahedron is related to the 13 -atom icosahedron. The new $\mathrm{M}_{78}$ global minimum is formed from this double icosahedron by the removal of two triangular faces.

All these new global minima, except that for $\mathrm{M}_{78}$, occur at values of $\rho$ where the basinhopping global optimization algorithm was not applied in the original study [7]. Instead, the location of global minima that are only optimal at intermediate $\rho$ values was dependent on the ingenuity of the authors (or lack of it in these cases) of that paper in constructing candidate structures.
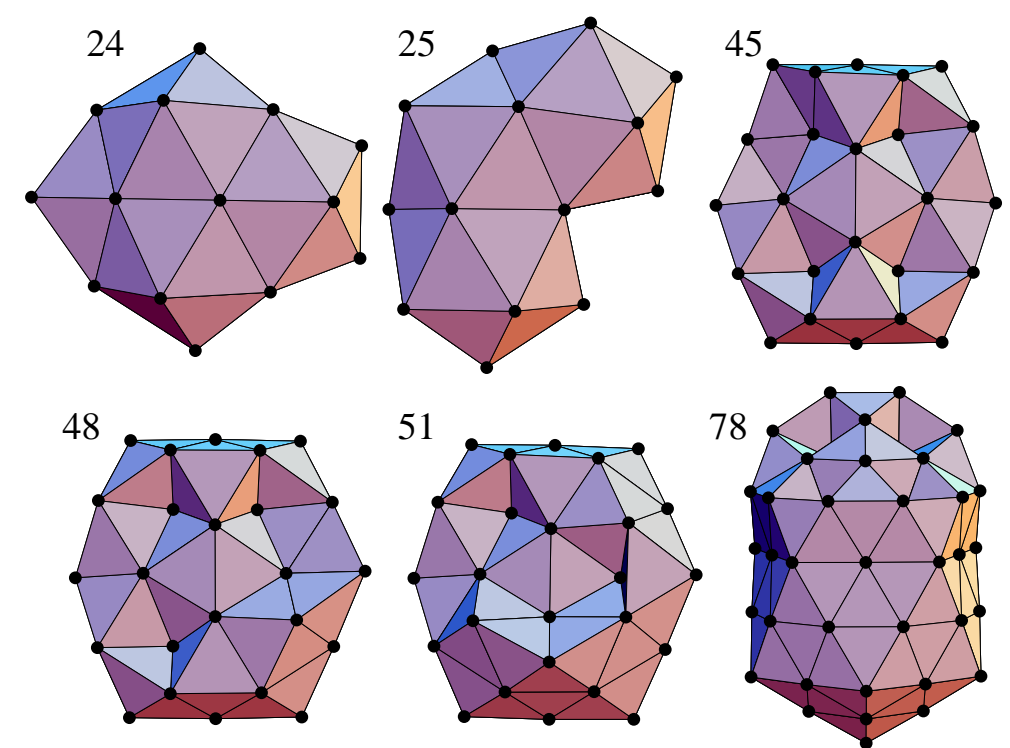

Figure 3: New Morse cluster global minima found during this research.

\section{Conclusion and future research}

We have shown that the two phase basin-hopping algorithm with shape-directing weighted diameter penalties is a highly effective and robust global optimization methodology for Morse clusters. A very limited parameter grid selected without detailed parameter tuning is sufficient to detect all known optimal clusters and also to find new ones.

However, we have also shown that there seems to exist a 'natural' choice of the weight parameters based on the cluster shape factors determined by the principle moments of inertia. One possible aim for future research is to investigate more sophisticated strategies than the current grid search that perhaps incorporate adaptive elements that exploit this connection. 
For example, the current weighted diameter penalty term is applied in a space-fixed, rather than molecule-fixed, coordinate system. Thus the corresponding compressional forces will be changed in the molecule-fixed system by simply rotating the cluster in the space fixedsystem, which does not seem appropriate if indeed we want to search over the space of cluster shapes. The insertion of an explicit rotation step after each local optimization to align the principle moment of inertia axes of the current iterate with the coordinate axes would more closely couple the diameter penalty to cluster shape during the course of the optimization.

Although the transformations of the Morse potential outlined here have been designed with clusters in mind, competing conformations in biomolecules are also likely to differ in their shape. Therefore, these methods are potentially transferable to the global optimization of biomolecules, such as in the protein folding problem. Even if the particularly penalty functions that we use here are not the most appropriate, the principles of our approach are still likely to apply: namely, the design of potential modifications that can discriminate between competing structure, which are then used in a series of optimization runs that favour different regions of shape space. Indeed, preliminary applications to protein-protein docking [2] have been encouraging.

\section{Acknowledgments}

J. P. K. Doye is grateful to the Royal Society for financial support.

R. H. Leary acknowledges support from National Science Foundation under Cooperative Agreement ACI9619020, and also the contributions of Kevin Chan of Harvard College who performed many of the MSBH runs, including those that found the new structure at $N=$ $78, \rho=6$.

M. Locatelli and F. Schoen acknowledge support from Progetto FIRB "Ottimizzazione Non Lineare su Larga Scala" and also the contribution of B. Addis, Università di Firenze for her support in the development of the software.

\section{References}

[1] V. I. Abkevich, A. M. Gutin, and E. I. Shakhnovich, Free energy landscape for protein folding kinetics: Intermediates, traps and multiple pathways in theory and lattice model simulations, J. Chem. Phys., 101, 6052-6062, (1994). 
[2] B. Addis and F. Schoen. A randomized global optimization method for protein-protein docking. Technical Report DSI 4-2003, Dipartimento di Sistemi e Informatica, Università degli Studi di Firenze, Firenze, (2003) (submitted).

[3] J. D. Bryngelson, J. N. Onuchic, N. D. Socci, and P. G. Wolynes, Funnels, pathways, and the energy landscape of protein folding: A synthesis, Proteins, 21, 167-195, (1995).

[4] The Cambridge Cluster Database, http://www-wales.ch.cam.ac.uk/CCD.html.

[5] J. P. K. Doye, The effect of compression on the global optimization of atomic clusters, Phys. Rev. E, 62, 8753-8761, (2000).

[6] J. P. K. Doye, M. A. Miller, and D. J. Wales, Evolution of the potential energy surface with size for Lennard-Jones clusters, J. Chem. Phys., 111, 8417-8428, (1999).

[7] J. P. K. Doye and D. J. Wales, Structural consequences of the range of the interatomic potential: A menagerie of clusters, J. Chem. Soc., Faraday Trans., 93, 4233-4244, (1997).

[8] J. P. K. Doye and D. J. Wales, Thermodynamics of global optimization, Phys. Rev. Lett., 80, 1357-1360, (1998).

[9] J. P. K. Doye, D. J. Wales, W. Branz, and F. Calvo, Modelling the structure of $\mathrm{C}_{60}$ clusters, Phys. Rev. B, 64, 235409, (2001).

[10] J. P. K. Doye, D. J. Wales, and S. I. Simdyankin, Global optimization and the energy landscapes of Dzugutov clusters, Faraday Discuss., 118, 159-170, (2001).

[11] C. A. Floudas, P. M. Pardalos, C.S. Adjiman, W. R. Esposito, Z. H, Gümüs, S. T. Harding, J. L. Klepeis, C. A. Meyer, and C. A. Schweiger, Handbook of Test Problems for Local and Global Optimization, Kluwer Academic, Dordrect, 1999.

[12] Z. Y. Guo and D. Thirumalai, Kinetics of protein-folding-nucleation mechanism, time scales, and pathways, Biopolymers, 36, 83-102, (1995).

[13] T. Kiefhaber, Kinetic traps in lysozyme folding, Proc. Natl. Acad. Sci. USA, 92, 90299033, (1995). 
[14] K. F. Lau and K. A. Dill, A lattice statistical-mechanics model of the conformational and sequence-spaces of proteins, Macromolecules, 22, 3986-3997, (1989).

[15] R. H. Leary, Global optimization on funneling landscapes, J. Global Optim., 18, 367$383,(2000)$.

[16] Y. Levy and O. M. Becker, Effect of conformational constraints on the topography of complex potential energy surfaces, Phys. Rev. Lett., 81, 1126, (1998).

[17] Y. Levy, J. Jortner, and O. M. Becker, Dynamics of hierarchical kinetics on the energy landscapes of hexapeptides, J. Chem. Phys., 115, 10533-10547, (2001).

[18] Z. Li and H. A. Scheraga, Monte-carlo-minimization approach to the multiple-minima problem in protein folding, Proc. Natl. Acad. Sci. USA, 84, 6611-6615, 1987.

[19] M.Locatelli, F.Schoen, Fast global optimization of difficult Lennard-Jones clusters, Computational Optimization and Applications, 21, 55-70 (2002)

[20] M.Locatelli, F.Schoen, Efficient algorithms for large scale global optimization: LennardJones clusters, to appear in Computational Optimization and Applications (2003)

[21] M. A. Miller, J. P. K. Doye, and D. J. Wales, Structural relaxation in Morse clusters: Energy landscapes, J. Chem. Phys., 110, 328-334, (1999).

[22] M. A. Miller and D. J. Wales, Energy landscape of a model protein, J. Chem. Phys., 111, 6610-6616, (1999).

[23] P. M. Morse, Diatomic molecules according to the wave mechanics. ii. Vibrational levels, Phys. Rev., 34, 57-64, (1929).

[24] P. N. Mortenson, D. J. Wales, and D. A. Evans, Energy landscapes of model polyalanines, J. Chem. Phys., 117, 1363-1376, (2002).

[25] J. A. Northby, Structure and bonding of Lennard-Jones clusters: $13 \leq n \leq 147, J$. Chem. Phys., 87, 6166-6177, (1987).

[26] S. E. Radford, C. M. Dobson, and P. A. Evans, The folding of hen lysozyme involves partially structured intermediates and multiple pathways, Nature, 358, 302-307, (1992). 
[27] C. Roberts, R. L. Johnston, and N. T. Wilson, A genetic algorithm for the structural optimization of Morse clusters, Theor. Chem. Acc., 104, 123-130, (2000).

[28] D. Romero, C. Barrón, and S. Gómez, The optimal geometry of Lennard-Jones clusters: 148-309, Comp. Phys. Comm., 123, 87-96, (1999).

[29] D. J. Wales and J. P. K. Doye, Global optimization by basin-hopping and the lowest energy structures of Lennard-Jones clusters containing up to 110 atoms, J. Phys. Chem. A, 101, 5111-5116, (1997).

[30] D. J. Wales and H. A. Scheraga, Global optimization of clusters, crystals and biomolecules, Science, 285, 1368-1372, (1999).

[31] M. D. Wolf and U. Landman, Genetic algorithms for structural cluster optimization, J. Phys. Chem. A, 102, 6129-6137, 1998. 